\title{
Charging and exciton-mediated decharging of metal nanoparticles in organic semiconductor matrices
}

Giovanni Ligorio, Marco Vittorio Nardi, Christos Christodoulou, Ileana Florea, Nicolas-Crespo Monteiro, Ovidiu Ersen, Martin Brinkmann, and Norbert Koch

Citation: Appl. Phys. Lett. 104, 163302 (2014); doi: 10.1063/1.4873347

View online: https://doi.org/10.1063/1.4873347

View Table of Contents: http://aip.scitation.org/toc/apl/104/16

Published by the American Institute of Physics

\section{Articles you may be interested in}

Metal nanoparticle mediated space charge and its optical control in an organic hole-only device Applied Physics Letters 108, 153302 (2016); 10.1063/1.4945710

Properties of hybrid organic-inorganic systems: Au nanoparticles embedded into an organic CuPc matrix Applied Physics Letters 97, 113103 (2010); 10.1063/1.3488809

Multilevel conductance switching in organic memory devices based on $\mathrm{AlQ}_{3}$ and $\mathrm{Al} / \mathrm{Al}_{2} \mathrm{O}_{3}$ core-shell nanoparticles

Applied Physics Letters 94, 173304 (2009); 10.1063/1.3123810

Plasmon-enhanced solar energy conversion in organic bulk heterojunction photovoltaics

Applied Physics Letters 92, 013504 (2008); 10.1063/1.2823578

Nonvolatile electrical bistability of organic/metal-nanocluster/organic system

Applied Physics Letters 82, 1419 (2003); 10.1063/1.1556555

Memory device applications of a conjugated polymer: Role of space charges

Journal of Applied Physics 91, 2433 (2002); 10.1063/1.1445281

\section{PHYSICS TODAY}

MANAGER'S GUIDE

WHITEPAPERS

\section{READ NOW}

PRESENTED BY

Accelerate R\&D with

Multiphysics Simulation $\subset \subset$ COSOL 


\title{
Charging and exciton-mediated decharging of metal nanoparticles in organic semiconductor matrices
}

\author{
Giovanni Ligorio, ${ }^{1, a)}$ Marco Vittorio Nardi, ${ }^{1, a), b)}$ Christos Christodoulou, ${ }^{1}$ Ileana Florea, ${ }^{2}$ \\ Nicolas-Crespo Monteiro, ${ }^{3, \mathrm{c})}$ Ovidiu Ersen, ${ }^{2}$ Martin Brinkmann, ${ }^{3}$ and Norbert Koch ${ }^{1,4, d)}$ \\ ${ }^{1}$ Institut für Physik, Humboldt-Universität zu Berlin, Brook-Taylor-Str. 6, 12489 Berlin, Germany \\ ${ }^{2}$ IPCMS, rue du loess 23, 67034 Strasbourg, France \\ ${ }^{3}$ Institut Charles Sadron CNRS, rue du loess 22, 67034 Strasbourg, France \\ ${ }^{4}$ Helmholtz-Zentrum Berlin für Materialien und Energie GmbH, Albert-Einstein-Str. 15, 12489 Berlin, \\ Germany
}

(Received 7 March 2014; accepted 14 April 2014; published online 23 April 2014)

\begin{abstract}
Gold nanoparticles (Au-NPs) were deposited on the surface of $n$ - and $p$-type organic semiconductors to form defined model systems for charge storage based electrically addressable memory elements. We used ultraviolet photoelectron spectroscopy to study the electronic properties and found that the Au-NPs become positively charged because of photoelectron emission, evidenced by spectral shifts to higher binding energy. Upon illumination with light that can be absorbed by the organic semiconductors, dynamic charge neutrality of the Au-NPs could be re-established through electron transfer from excitons. The light-controlled charge state of the AuNPs could add optical addressability to memory elements. (c) 2014 AIP Publishing LLC. [http://dx.doi.org/10.1063/1.4873347]
\end{abstract}

The combination of organic semiconductors (both small molecules and polymers) with metal nanoparticles to a hybrid material is increasingly attracting interest for various applications. Light emitting diodes (LEDs) and photovoltaic cells may exploit optical and plasmonic functionalities of metal nanoparticles (NPs). ${ }^{1-3}$ Field effect transistors (FETs) and non-volatile memory elements could employ NPs as charge storage centers, where depending on their charge state, the conductivity of the hybrid material may be modified by several orders of magnitude, allowing the devices to manifest a bistable electrical behavior. ${ }^{4-6}$ The metal NP charge state change is usually achieved by driving an appropriate current through the device.,

Recently, hybrid FETs with gold NPs (Au-NPs), in part functionalized with a photoresponsive azobenzene derivate, were reported to provide optical switching capability to the device besides the electric control through the gate electrode. ${ }^{5}$ Earlier work suggested that charge transfer between diindenoperylene and Au-NPs can be optically induced, without the need for NP functionalization. ${ }^{9}$ However, the structural and morphological complexity of that system, i.e., multicrystalline organic films with two textures and diffusion of Au-NPs-particularly at grain boundaries, precluded clear-cut identification of this mechanism beyond any level of doubt.

In this work, we investigated optically induced decharging of Au-NPs with two of the most widely used organic semiconductors, i.e., the electron transport materials aluminum tris(8-hydroxyquinoline) $\left(\mathrm{Alq}_{3}\right)$ and the hole transport

\footnotetext{
${ }^{a)}$ G. Ligorio and M. Vittorio Nardi contributed equally to this work.

b)nardi@physik.hu-berlin.de

${ }^{c)}$ Present address: Université de Lyon, F-42023 Saint-Etienne, France; CNRS, UMR 5516, Laboratoire Hubert Curien, 18 rue Pr. Lauras F-42000 Saint-Etienne; Université de Saint-Etienne, Jean-Monnet, F-42000 SaintEtienne.

d)norbert.koch@physik.hu-berlin.de
}

material 4,4-bis[N-(1-naphthyl)-N-phenyl-amino]diphenyl $(\alpha-\mathrm{NPD})$. The choice of these two materials is justified by their known simple morphology in films, i.e., smooth amorphous films formed upon thermal evaporated; ${ }^{10,11}$ and their complementary ability to transport electrons or holes. Au was chosen as metal for NPs because of its frequent use in organic electronic devices. With these material combinations, we were able to grow well defined hybrid structures, notably with defined Au-NP position and size distribution in the organic matrices. This enabled the unequivocal identification of exciton-mediated decharging of the Au-NPs, which might be useful to add optical functionality to hybrid memory elements.

UPS experiments were performed at the end station SurICat (beamline PM4) of the storage ring BESSY II (Berlin, Germany). $\mathrm{Alq}_{3}$ and $\alpha$-NPD (Aldrich) were sublimated in situ in ultrahigh vacuum (UHV, pressure below $5 \times 10^{-9}$ millibars) conditions from a resistively heated pinhole source onto indium tin oxide (ITO) coated glass. The ITO substrates were previously cleaned by sequential sonication in acetone and isopropyl alcohol. The nominal thickness for both films was $400 \AA$ (evaporation rate ca. $0.15 \AA \mathrm{s}^{-1}$ ). The samples were transferred to the analysis chamber (base pressure $5 \times 10^{-10}$ millibars) without breaking UHV for the UPS characterization. The photon energy was set to $35 \mathrm{eV}$, and the resolution was $150 \mathrm{meV}$. UPS measurements were always performed under two different conditions: dark, i.e., only synchrotron radiation (for UPS) was irradiating the sample, and in light, i.e., the sample was illuminated with an external light source during the UPS measurement. We used a $405 \mathrm{~nm}$ wavelength solid-state laser; this wavelength can be absorbed by both $\alpha$-NPD and $\mathrm{Alq}_{3} .{ }^{10}$ The laser beam was spread over the whole sample with a quartz lens, the power density was $0.02 \mathrm{~mW} \mathrm{~cm}^{-2}$. No damage of the samples was observed. Au-NPs were evaporated in a stepwise manner onto the organic films using an electron beam evaporator 
(nominal evaporation rate $0.01 \AA^{-1}$; the nominal mass-thickness $\Theta$ was monitored with a quartz crystal microbalance). After each deposition step, UPS spectra of organic/Au-NPs were acquired in dark and in light.

The sample morphology was investigated by transmission electron microscopy (TEM). Samples fully equivalent to those investigated by UPS $\left(\mathrm{Alq}_{3} / \mathrm{Au}-\mathrm{NPs}\right.$ and $\alpha$-NPD/Au-NPs, nominal Au mass-thickness $\Theta_{\mathrm{Au}}=0.3 \AA, 1.0 \AA, 3.0 \AA$ ) were prepared on spin coated poly(3,4-ethylenedioxythiophene): poly(styrenesulfonic acid) (PEDOT:PSS, AI4083 purchased from Heraeus-Clevios) as interlayer between organic and ITO substrates. Additionally, $400 \AA$ of the respective organic material were evaporated on the top of the organic/M-NPs surface in order to protect the sample for the TEM ex-situ characterization. For TEM analysis, the samples were coated first with a thin amorphous carbon layer and after dissolution of the PEDOT:PSS in a diluted aqueous HF solution ( $<2$ wt. $\%)$, the thin films containing the hybrid layer were recovered on TEM copper grids. Bright field TEM and electron tomography were performed [spherical aberration $(\mathrm{Cs})$ probe corrected JEOL $2100 \mathrm{~F}$ ] in order to obtain a $3 \mathrm{D}$ reconstructured image of the Au-NP distribution (position and size) in the organic matrix $^{12-14}$ and thus to examine the correlation between $\mathrm{Au}-\mathrm{NPs}$ size and spatial distribution with respect to the nominal mass-thickness of gold deposited on the organic surface.

Figure 1 shows two samples with different nominal evaporated $\mathrm{Au}$ mass-thickness $\left(\Theta_{\mathrm{Au}}\right)$ deposited on $\mathrm{Alq}_{3}$. Figs. 1(a) and 1(b) are bright field TEM images ( $x y$-plane) of samples with $\Theta_{\mathrm{Au}}=0.3 \AA$ and $3.0 \AA$, respectively. In the sub-nanometer thickness regime, Au does not grow homogeneously in a compact metallic film but forms NPs, ${ }^{15}$ as confirmed by the figure. To investigate whether interdiffusion of NPs into the organic bulk is significant, a series of images ( $x z$-planes) with different tilt angles was acquired. Figs. 1(c) and $1(\mathrm{~d})$ show the projection of cross sectional images ( $x z$-plane) that were reconstructed from the tilt series for both samples. The images show clearly that Au-NPs lie in one plane orthogonal to the cross section plane, i.e., diffusion is not an issue, and no percolation of Au-NPs within the plane occurs, i.e., the NPs are laterally separated from each other. Statistical analysis of the samples shows that $\Theta_{\mathrm{Au}}$ is correlated with the average particle diameter $\left(d_{\mathrm{Au}-\mathrm{NPs}}\right)$, the particle-particle distance calculated with a statistical autocorrelation function $\left(\lambda_{\mathrm{NP}-\mathrm{NP}}\right.$, autocorrelation peak defined only for sample $\Theta_{\mathrm{Au}}=3.0 \AA$ ), and the number of NPs per surface unit area $\left(\sigma_{\mathrm{Au}-\mathrm{NP}}\right)$. Figs. 1(e) and 1(f) show the histograms of Au-NPs dimension populations. The parameters extracted from statistical analysis are summarized in Table I, including data from one more sample with $\Theta_{\mathrm{Au}}=1 \AA$.

Fully analogous results were found for $\alpha-\mathrm{NPD} / \mathrm{Au}-\mathrm{NPs}$. Likewise, Au does not grow as a homogeneous film on $\alpha$ NPD in the sub-nanometer thickness regime and the NP layer is formed right at the organic surface.

Figures 2(a) and 2(b) show the evolution of the UPS spectra for an increasing amount of $\mathrm{Au}$ on $\mathrm{Alq}_{3}$ and $\alpha$-NPD, respectively. Spectra were measured in dark (black thick line) and in light, i.e., with additional laser illumination (blue thin line); the energy shift between the two conditions is indicated next to the spectra. Note that the laser power $(0.02 \mathrm{~mW}$ $\mathrm{cm}^{-2}$ ) was always sufficient to saturate the shift (i.e., additional increase of laser power did not result in a further shift).

The spectra of the pristine molecular films $\left(\Theta_{\mathrm{Au}}=0.0 \AA\right)$ are shown at the bottom. The highest occupied molecular orbital (HOMO) onset is found at $1.9 \mathrm{eV}$ and $1.2 \mathrm{eV}$ below $\mathrm{E}_{\mathrm{F}}$ for $\mathrm{Alq}_{3}$ and $\alpha$-NPD, respectively, and there is no energy shift or spectral shape change when measured in dark and in light. Once Au-NPs are on the surface, the spectral shape in the valence region significantly changes, and the formerly sharp peaks related to the molecular materials can barely be discerned. For increasing $\Theta_{\mathrm{Au}}$, the spectra lose the typical molecular fingerprints, and even the onset of the HOMO is not well defined anymore. The $\mathrm{Au}$ Fermi-edge appears from $\Theta_{\mathrm{Au}}=0.9 \AA$ onwards (see inset in Fig. 2(b), evidencing that (some of) the NPs are metallic. Since no continuous metal film has formed at this $\Theta_{\mathrm{Au}}$, the number of $\mathrm{Au}$ atoms per cluster is high enough to render them metallic. At this coverage, the spectra of both systems are strikingly similar and they are dominated by emission from $\mathrm{Au}$ (essentially two broad
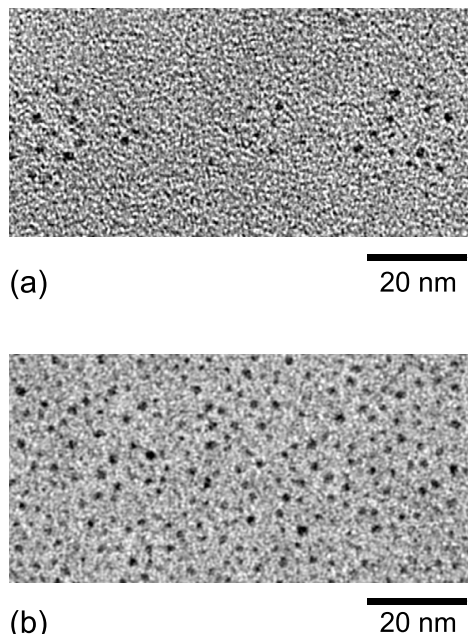

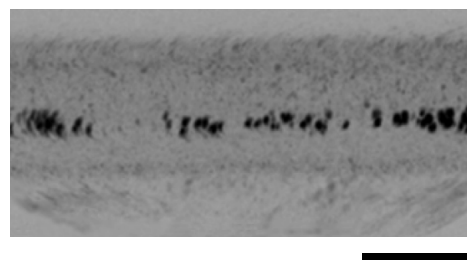

(c)

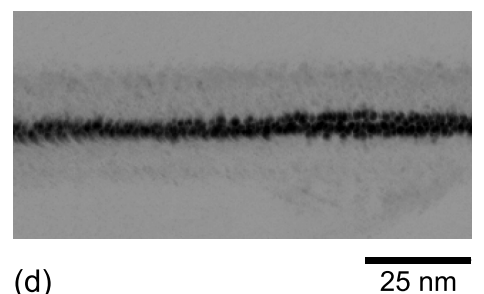

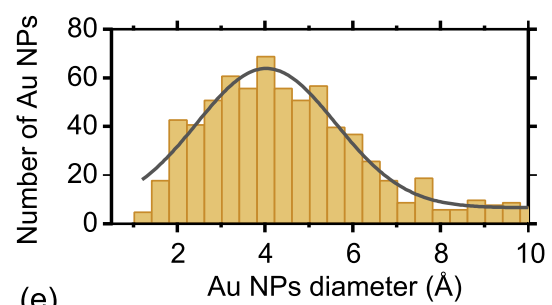

(e)

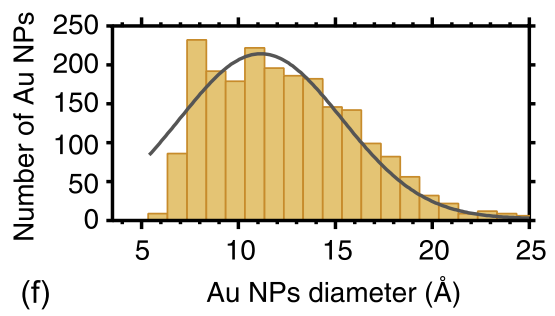

FIG. 1. Comparison of Au-NPs/Alq 3 samples with different nominal Au mass-thicknesses $\left(\Theta_{\mathrm{Au}}=0.3 \AA\right.$ on the top, and $3.0 \AA$ at the bottom). (a) and (b) are top view (xy-plane) TEM images. (c) and (d) are cross sectional TEM 3D tomography images (projection along $x z$-plane) of the $0.3 \AA$ and $3.0 \AA$ samples, respectively. (e) and (f) are histograms of the Au-NP size populations. 
TABLE I. TEM statistical analysis results regarding average particle diameter $\left(d_{\text {Au-NPs }}\right)$, particle-particle distance $\left(\lambda_{\text {NP-NP }}\right)$, and number of NPs per surface unit $\left(\sigma_{\mathrm{Au}-\mathrm{NP}}\right)$ for samples with different nominal Au mass-thicknesses $\Theta_{\mathrm{Au} \text {. }}$

\begin{tabular}{lrcc}
\hline \hline$\Theta_{\mathrm{Au}}(\AA)$ & $d_{\mathrm{Au}-\mathrm{NPs}}(\AA)$ & $\lambda_{\mathrm{NP}-\mathrm{NP}}(\AA)$ & $\sigma_{\text {Au-NPs }}\left(\mathrm{NPs} / 100 \mathrm{~nm}^{2}\right)$ \\
\hline 0.3 & $4 \pm 1$ & $\ldots$ & $>1$ \\
1.0 & $8 \pm 1$ & $\ldots$ & 2 \\
3.0 & $11 \pm 1$ & 33 & 8 \\
\hline \hline
\end{tabular}

features at $4 \mathrm{eV}$ and $6 \mathrm{eV}$, related to the $\mathrm{Au} 5 \mathrm{~d}_{3 / 2}$ and $\mathrm{Au} 5 \mathrm{~d}_{5 / 2}$ levels ${ }^{16}$ ).

In dark measurement conditions, for both systems, the molecular valence structures are shifted towards higher binding energy (by up to $1.0 \mathrm{eV}$ ) for $\Theta_{\mathrm{Au}}=0.1 \AA, 0.3 \AA$, and $0.4 \AA$. As revealed by TEM analysis, the Au-NPs are well separated. Due to the photoemission process itself, the Au-NPs become positively charged and charge neutralization by electrons from the substrate is not efficient because the hole state on the Au-NP corresponds to a deep hole trap in the organic matrix. The positive space charge at the surface thus reduces the kinetic energy of the photoelectrons, shifting the UPS spectra toward higher binding energy. This charging mechanism is schematically depicted in Fig. 2(c).

In light (for $\Theta_{\mathrm{Au}}=0.1 \AA, 0.3 \AA$, and $0.4 \AA$ ), i.e., with additional laser illumination, the spectra are shifted towards lower binding energy compared to those measured in dark. This back-shift brings particular molecular emission features, e.g., at $2.5 \mathrm{eV}$ and $5 \mathrm{eV}$ for $\mathrm{Alq}_{3}$ and at $4 \mathrm{eV}$ and $6 \mathrm{eV}$ for $\alpha-N P D$, to the same energy position as was observed without Au-NPs. Obviously, the illumination with visible light eliminates the space charge on the Au-NPs, i.e., the NPs become decharged due to light absorption by the organic matrix, as shown in the schematic of Fig. 2(d). Upon illumination, excitons are created in the organic matrix; electron transfer from the molecular lowest unoccupied molecular orbital (LUMO) to the NPs is allowed because of the favorable energy level alignment. Now there is a dynamic equilibrium between the charging of NPs due to the photoemission process and the decharging, resulting from the exciton dissociation at the organic/Au-NPs interface; the net surface space charge approaches zero if the light intensity is appropriately adjusted (as in the case presented here).

One note regarding the spectral broadening upon deposition of $\mathrm{Au}$ on a-NPD and $\mathrm{Alq}_{3}$ : In our experiments, the $\mathrm{Au}$ mass-thickness was estimated with a quartz crystal microbalance, therefore, there might be slight differences between the actual $\mathrm{Au}$ amounts on both organic surfaces at the same nominal thickness. In addition, the lateral distribution of $\mathrm{Au}$ clusters and atoms, too small to be individually resolved by TEM, may be inhomogeneous, therefore, leading to a more pronounced spectral broadening for $\mathrm{Alq}_{3}$.

In summary, optically induced electron transfer from organic semiconductors to positively charged Au-NPs was demonstrated. TEM studies manifested the defined growth of $\mathrm{Au}-\mathrm{NPs}$ on $\mathrm{Alq}_{3}$ and $\alpha$-NPD surfaces, which allows unambiguously evidencing the decharging mechanism. In our experiments, the photoelectron emission from Au-NPs created the surface charge on the Au-NPs, and exciton generation in the organic semiconductors was facilitated by laser irradiation. By appropriate selection of the laser intensity, the charge on $\mathrm{Au}$-NPs could be completely removed, i.e., dynamic electronic equilibrium was established. This phenomenon might (a)

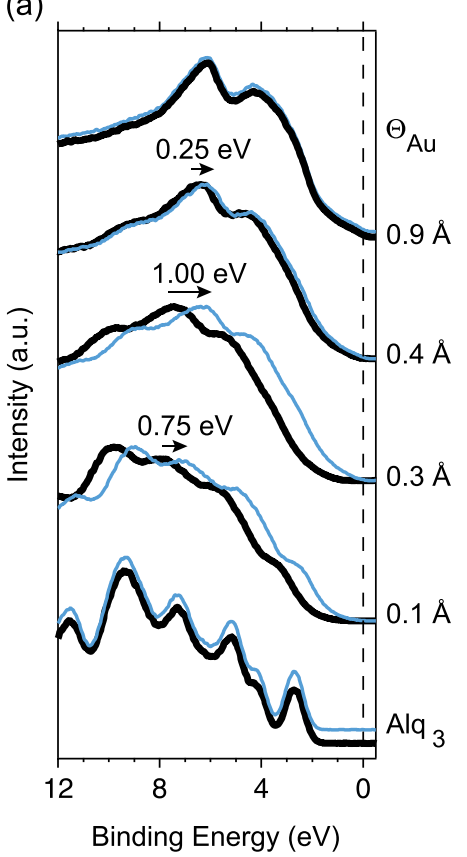

(b)

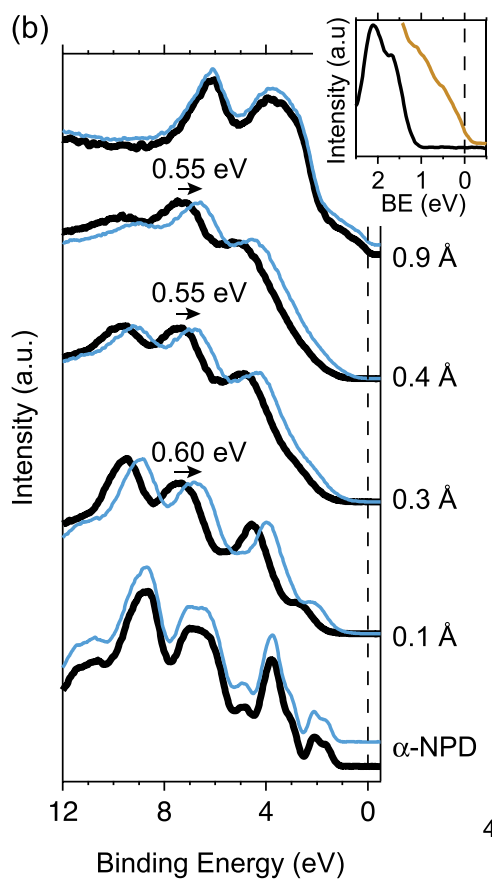

(c)
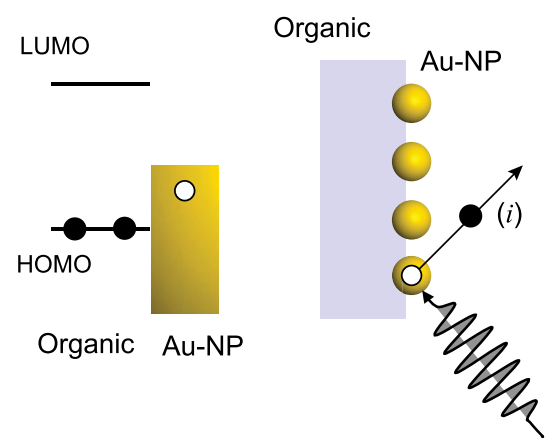

(d)

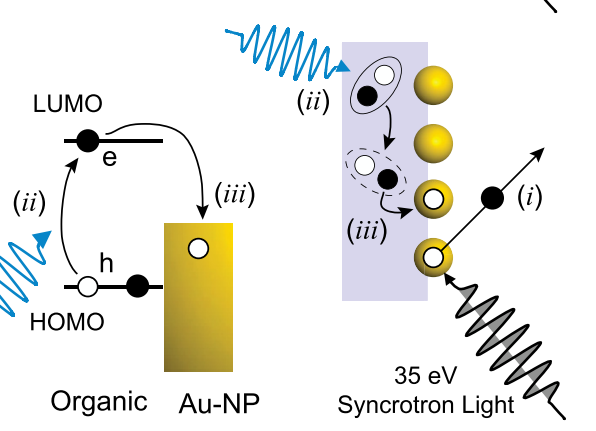

FIG. 2. UPS spectra for $\mathrm{Alq}_{3} / \mathrm{Au}-\mathrm{NPs}$ in (a) and $\alpha$-NPD/Au-NPs in (b) for increasing amount of Au ( $\left.\Theta_{\text {Au }}\right)$. The measurements were taken without (black thick lines) and with external laser (blue thin lines). The inset in (b) shows the Fermi-edge of the metal covered $\alpha$-NPD film compared to the spectrum of the pristine molecular film. (c) and (d) show schematic energy level diagrams and involved processes for the system without and with laser, respectively. (i) electron photoemission with consequent positive charging of NPs, (ii) laser light absorption with exciton formation, (iii) electron transfer from the molecular LUMO levels to the Au-NPs (decharging). 
be exploited in hybrid organic/metal-NPs devices, where optical conductivity switching could be used as in addition to electrical addressing.

The authors gratefully acknowledge financial support from the European Commission FP7 Project HYMEC (Grant No. 263073). C. Blank and M. Schmutz are acknowledged for technical support with transmission electron microscopy.

${ }^{1}$ D. Liu, M. Fina, L. Ren, and S. S. Mao, Appl. Phys. A 96, 353 (2009).

${ }^{2}$ J.-L. Wu, F.-C. Chen, Y.-S. Hsiao, F.-C. Chien, P. Chen, C.-H. Kuo, M. H. Huang, and C.-S. Hsu, ACS Nano 5, 959 (2011).

${ }^{3}$ M.-C. Chen, Y. Yang, S. Chen, J. Li, M. Aklilu, and Y. Tai, ACS Appl. Mater. Interfaces 5, 511 (2013).

${ }^{4}$ L. Ma, S. Pyo, J. Ouyang, Q. Xu, and Y. Yang, Appl. Phys. Lett. 82, 1419 (2003).

${ }^{5}$ C. Raimondo, N. Crivillers, F. Reinders, F. Sander, M. Mayor, and P. Samorì, Proc. Natl. Acad. Sci. U.S.A. 109, 12375 (2012); S. Han, Y. Zhou, Q.-D. Yang, C.-S. Lee, and V. A. L. Roy, Part. Part. Syst. Charact 30, 599 (2013).
${ }^{6}$ M. Kang, K.-J. Baeg, D. Khim, Y.-Y. Noh, and D.-Y. Kim, Adv. Funct. Mater. 23, 3503 (2013).

${ }^{7}$ L. D. Bozano, B. W. Kean, M. Beinhoff, K. R. Carter, P. M. Rice, and J. C. Scott, Adv. Funct. Mater. 15, 1933 (2005).

${ }^{8}$ J. C. R. Scott and L. D. D. Bozano, Adv. Mater. 19, 1452 (2007).

${ }^{9}$ N. Koch, A. C. Dürr, J. Ghijsen, R. L. Johnson, J.-J. Pireaux, J. Schwartz, F. Schreiber, H. Dosch, and A. Kahn, Thin Solid Films 441, 145 (2003).

${ }^{10}$ M. Brinkmann, G. Gadret, M. Muccini, C. Taliani, N. Masciocchi, and A. Sironi, J. Am. Chem. Soc. 122, 5147 (2000).

${ }^{11}$ L. S. Hung and C. H. Chen, Mater. Sci. Eng. R Rep. 39, 143 (2002).

${ }^{12}$ W. O. O. Saxton, W. Baumeister, and M. Hahn, Ultramicroscopy 13, 57 (1984).

${ }^{13}$ I. Florea, O. Ersen, C. Hirlimann, L. Roiban, A. Deneuve, M. Houllé, I. Janowska, P. Nguyen, C. Pham, and C. Pham-Huu, Nanoscale 2, 2668 (2010).

${ }^{14}$ L. Roiban, L. Hartmann, A. Fiore, D. Djurado, F. Chandezon, P. Reiss, J.F. Legrand, S. Doyle, M. Brinkmann, and O. Ersen, Nanoscale 4, 7212 (2012).

${ }^{15}$ A. C. Dürr, N. Koch, M. Kelsch, A. Rühm, J. Ghijsen, R. Johnson, J.-J. Pireaux, J. Schwartz, F. Schreiber, H. Dosch, and A. Kahn, Phys. Rev. B 68, 115428 (2003).

${ }^{16}$ H. Roulet, J.-M. Mariot, G. Dufour, and C. F. Hague, J. Phys. F Met. Phys. 10, 1025 (1980). 